\title{
Additional Value of FDG PET and Resting State-functional MRI for the Assessment of Consciousness Disorders in Hypoglycemia-induced Coma
}

\author{
Bund $\mathbf{C}^{1}$, Roquet $\mathbf{D}^{2}$, Noblet $\mathbf{V}^{2}$, Schenck-Dhif $\mathbf{M}^{3}$, Schneider $\mathbf{F}^{3}$, Kremer $\mathbf{S}^{2,4,5}$ and Namer $\mathbf{I J}^{1,2,5^{*}}$ \\ ${ }^{1}$ Department of Biophysics and Nuclear Medicine, University Hospital of Strasbourg, Strasbourg, France \\ ${ }^{2}$ ICube, University of Strasbourg / CNRS (UMR 7357), Strasbourg, France \\ ${ }^{3}$ Medical Intensive Care Unit, University Hospital of Strasbourg, Strasbourg, France \\ ${ }^{4}$ Radiology and Hospital Hautepierre Service, University Hospital of Strasbourg, Strasbourg, France \\ ${ }^{5}$ Translational Medicine Federation Strasbourg (WFSW), Faculty of Medicine, Strasbourg, France
}

${ }^{*}$ Corresponding author: Izzie-Jacques Namer, Biophysics Department and Nuclear Medicine, University Hospital of Strasbourg, Hospital de Hautepierre, 1, avenue Molière, 67098 Strasbourg Cedex 09, France, Tel: +33388127550; Fax: +33388128342; E-mail: Izzie.Jacques.NAMER@chru-strasbourg.fr

Received date: January 28, 2016; Accepted date: February 10, 2016; Published date: February 17, 2016

Copyright: $(2016$ Bund C, et al. This is an open-access article distributed under the terms of the Creative Commons Attribution License, which permits unrestricted use, distribution, and reproduction in any medium, provided the original author and source are credited.

\begin{abstract}
Background: Consciousness disorders in brain-injured patients are sometimes complicated to understand. The accuracy of the distinction between coma, vegetative state, minimally conscious state and locked-in syndrome can be improved by using functional imaging modalities, which is important because of the therapeutic impact.

Case presentation: We report a case of a 34 years old diabetic Caucasian woman whose glycemia had been previously tightly controlled with an insulin pump and who developed a profound coma overnight (spontaneous ventilation and Glasgow score 6) with transient ocular revulsions and intermittent crawling movements triggered by noxious stimuli at admission. At the $3^{\text {rd }}$ day, 18F-fluorodeoxyglucose (FDG) PET and resting-state functional MRI (rs-fMRI) were performed when the patient spontaneously opened her eyes, but no evidence of awareness of the environment could be obtained. Awareness recovered progressively and follow-up imaging was performed on the $7^{\text {th }}$ day when the patient presented only certain cognitive disorders. We demonstrated that rs-fMRI and FDG PET are non-invasive imaging tools that are helpful in the assessment of consciousness levels and residual cognitive disorders in patients with metabolic severe coma.
\end{abstract}

Keywords: Coma; Hypoglycemia; Metabolic coma; PET; FDG; fMRI

\section{Introduction}

Consciousness disorders in brain-injured patients are sometimes complicated to understand. In fact, it could be a border zone between awareness and consciousness. The accuracy of the distinction between coma, vegetative state, minimally conscious state and locked-in syndrome can be improved by using functional imaging modalities, which is important because of the therapeutic impact. Many functional MRI and FDG PET data have been published over the last few years and have helped us in the understanding of consciousness disorders [1].

The case presented illustrates the additional value of rs-fMRI and FDG PET for the assessment of consciousness disorders in hypoglycemia-induced coma.

\section{Case}

Our patient was a 34 years old diabetic Caucasian woman whose glycemia had been previously tightly controlled with an insulin pump. She's diabetic since her 8th birthday and has a diabetic retinopathy. There were no other significant medical histories.

She developed a profound coma overnight (spontaneous ventilation and Glasgow score 6) with transient ocular revulsions and intermittent crawling movements triggered by noxious stimuli at admission (Table
1). She was transferred in a medical intensive care unit, where she was intubated. It's important to precise that she never required sedation or anesthesia. Cerebral CT at admission didn't show any remarkable signs. After treatment by glucose infusion and insulin (Table 2) she stayed in a prolonged coma, with a bilateral, symmetric and diffuse but slow activity on EEG, which is why she was included in an imaging protocol for patients presenting prolonged coma.

So, at the $3^{\text {rd }}$ day, FDG PET and rs-fMRI were performed when the patient spontaneously opened her eyes, but no evidence of awareness of the environment could be obtained.

A morphological brain MRI (including sagittal T1, axial T2, axial and coronal FLAIR, axial gradient echo T2 and diffusion weighted MR images) with rs-fMRI (405 whole-brain $\mathrm{T} 2{ }^{*}$-weighted echo planar images) was first performed. The first five images of rs-fMRI were removed to account for $\mathrm{T} 1$ partial saturation and the 400 remaining images were then motion corrected using SPM8 (Welcome Department of Cognitive Neurology, London, UK). Functional connectivity was analyzed using independent component analysis (FMRLAB toolbox 2.3, Swartz Center for Computational Neuroscience, San Diego, CA, USA) after reduction of the dimensionality from 400 to 250 by principal component analysis. Functional connectivity networks maps were manually selected following validated criteria [2] and classified according to the atlas from Kalcher et al. [3]. 
Citation: Bund C, Roquet D, Noblet V, Schenck-Dhif M, Schneider F (2016) Additional Value of FDG PET and Resting State-functional MRI for the Assessment of Consciousness Disorders in Hypoglycemia-induced Coma. J Neurol Neurophysiol 7: 355. doi: $10.4172 / 2155-9562.1000355$

Page 2 of 4

\begin{tabular}{|c|c|c|c|c|}
\hline & Initial management & $\begin{array}{l}\text { Arrival in medical intensive care } \\
\text { unit }\end{array}$ & Day 3: First FDG PET & Day 7: Second FDG PET \\
\hline Glasgow score & 6 & 3 & $\begin{array}{l}\text { no evidence of awareness of } \\
\text { the environment }\end{array}$ & disorientation in time and space \\
\hline Glycemia (mmol/L) & $1.78 a$ & 6.39 & 5.56 & 4.44 \\
\hline Blood pressure $(\mathrm{mmHg})$ & $80 / 50$ & $97 / 60$ & $108 / 62$ & $99 / 60$ \\
\hline $\begin{array}{l}\text { Cardiac frequency (beats per } \\
\text { minute) }\end{array}$ & 134 & 134 & 109 & 77 \\
\hline $\mathrm{SpO} 2(\%)$ & - & $97 \mathrm{~b}$ & $97 \mathrm{~b}$ & 100 \\
\hline $\mathrm{T}\left({ }^{\circ} \mathrm{C}\right)$ & - & 37 & 37.3 & 37.1 \\
\hline Potassium (mmol/L) & - & 3.74 & 4.41 & 3.87 \\
\hline CPK (UI/L) & - & $719 c$ & 110 & 56 \\
\hline Creatinine $(\mu \mathrm{mol} / \mathrm{L})$ & - & 68.8 & 76.2 & 68.9 \\
\hline Hemoglobin (g/dL) & - & 11.7 & 10.3 & $8.7 \mathrm{~d}$ \\
\hline Blood platelets (G/L) & - & 186 & 267 & 316 \\
\hline Leucocyte (G/L) & - & 5.1 & 6.3 & 4.91 \\
\hline
\end{tabular}

Table 1: Vitals and blood sampling results during hospitalization at key moments (a. Capillary blood glucose, b. Tracheal intubation + oxygen therapy, c. CPK (creatine phosphokinase) were elevated, up to $2718 \mathrm{UI} / \mathrm{L}$ in the first 24-hours, suggesting that coma was prolonged before the patient was found, $d$. During the hospitalization, an anemia was discovered on blood sample linked to an iron-deficiency anemia, which was then supplemented).

\begin{tabular}{|l|l|l|}
\hline Initial management & Reanimation & Output medical treatment \\
\hline $\begin{array}{l}\text { Subcutaneous insulin pump: insulin } \\
\text { glargine }\end{array}$ & $\begin{array}{l}\text { Glucose infusion } 30 \% \\
\text { then Perinutriflex } 1250 \mathrm{~mL} / 24 \mathrm{~h}\end{array}$ & $\begin{array}{l}\text { Intravenous rapid insulin infusion by an electric syringe pump in } \\
\text { doses controlling glycemia at } 4.4-8.3 \mathrm{mmol} / \mathrm{l}\end{array}$ \\
\hline Potassium chloride: $600 \mathrm{mg}$ & $\begin{array}{l}\text { Intravenous rapid insulin infusion by an electric syringe } \\
\text { pump in doses controlling glycemia at 4.4-8.3 mmol// }\end{array}$ & Potassium chloride: $1200 \mathrm{mg}$ \\
\hline Nicotine patch & Amoxicillin and clavulanic acid: $3 \mathrm{~g}$ & Amoxicillin and clavulanic acid: $3 \mathrm{~g}$ \\
\hline & $\begin{array}{l}\text { Low molecular weight heparin in thromboembolism } \\
\text { prevention }\end{array}$ & Vitamins B1 and B6 \\
\hline & Esomeprazole: $40 \mathrm{mg}$ & Ferrous sulphate \\
\hline
\end{tabular}

Table 2: Insulin dosing and medications prescribed to the patient.

While no abnormalities were seen on morphological MRI, four functional connectivity networks were observed on rs-fMRI: occipital, frontal and temporal networks, and left latero-fronto-parietal network (FPN). The internal awareness network, also called default mode network (DMN) was not identified (Figure 1A). Brain FDG PET showed hypometabolism of bilateral precuneus, left pre- and postcentral regions, left insula, left temporal regions and bilateral occipital regions (the patient's eyes were closed) (Figure 2B).

Awareness recovered progressively and follow-up imaging was performed on the 7 day when the patient presented only certain cognitive disorders, in particular disorientation in time and space (with a mini mental state examination at 15/30). The morphological MRI was normal. On rs-fMRI, five functional connectivity networks were observed including the DMN and the dorsal attentional system (C.04 and C.10 of the Kalcher's atlas, respectively), that were absent at the first MRI session (Figure 1B). FDG PET showed a marked improvement of previously observed metabolic abnormalities as only the left temporal pole remained hypometabolic (Figure 2C).

Restoration of spatial and temporal orientations, of normal cognition required an additional week of supportive care. 
Citation: Bund C, Roquet D, Noblet V, Schenck-Dhif M, Schneider F (2016) Additional Value of FDG PET and Resting State-functional MRI for the Assessment of Consciousness Disorders in Hypoglycemia-induced Coma. J Neurol Neurophysiol 7: 355. doi: $10.4172 / 2155-9562.1000355$

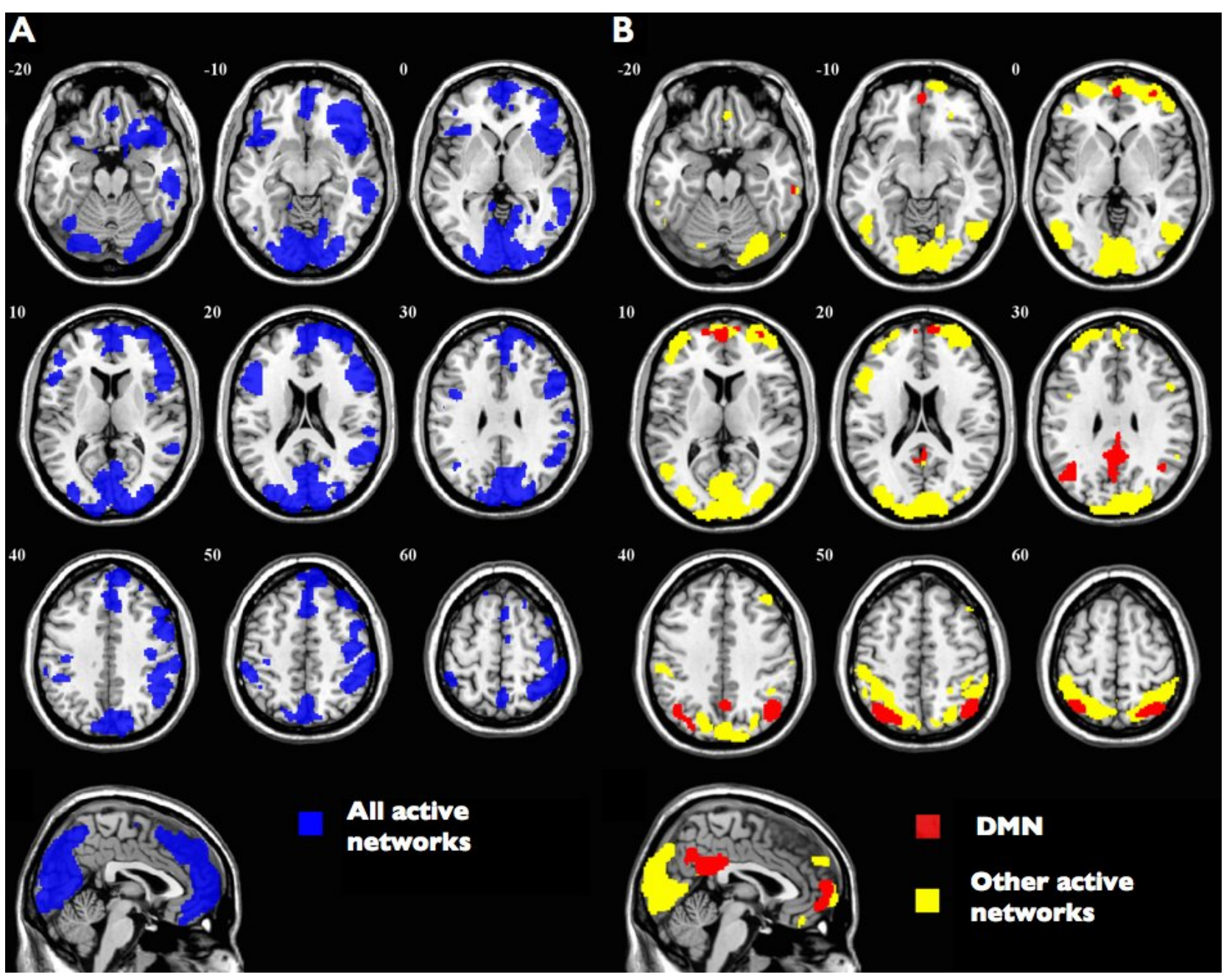

Figure 1: Networks from rs-fMRI acquisition during (A) and after (B) the hypoglycemic coma episode. During the coma episode (A) four functional connectivity networks (in blue) were observed on rs-fMRI (occipital, frontal, and temporal networks) including the left-FPN but not the DMN. After the coma episode (B), we observed four functional connectivity networks observed previously (in yellow) as well as the DMN and the dorsal attentional system (in red), which were absent at the first fMRI session.

\section{Discussion}

Detection of DMN was linked to better awareness. In fact, the last few years two main functional connectivity networks have been identified: the external awareness network (FPN) which subserves consciousness of the environment and the internal awareness network or default mode network (DMN) that appears to be related to selfrelated processes [4]. rs-fMRI studies have pointed out the importance of the DMN to distinguish coma, vegetative state, minimally conscious state and locked in syndrome as DMN connectivity correlates with the degree of consciousness [5].

FDG PET can demonstrate the relationship between functional activity and glucose energy metabolism in the central nervous system [6-9], as approximately $75 \%$ of brain's energy consumption goes into signal transmission, while the remaining $25 \%$ serves to maintain basic cellular activity [10]. FDG PET has proven able to distinguish the different levels of consciousness disorders by showing that glucose metabolism in the frontal and parietal cortex correlates with behavioral scores [11]. In our case, although rs-fMRI was considered as normal at the second session, FDG PET still showed left temporal hypometabolism that could explain the persistence of some cognitive disorders.

So, FDG PET could be used to see if patients would be emerging from minimally conscious state, which is not easy to do at bedside clinical examination. Recovery of the DMN metabolic activity was studied in severe brain damaged patients [12] and also in traumatic diffuse brain injuries [13]. Both studies showed that FDG PET was useful to distinguish between the different states of coma by studying hypometabolism. rs-fMRI showed similar results, although it seems to be less sensitive [12]. 
Citation: Bund C, Roquet D, Noblet V, Schenck-Dhif M, Schneider F (2016) Additional Value of FDG PET and Resting State-functional MRI for the Assessment of Consciousness Disorders in Hypoglycemia-induced Coma. J Neurol Neurophysiol 7: 355. doi: $10.4172 / 2155-9562.1000355$

Page 4 of 4

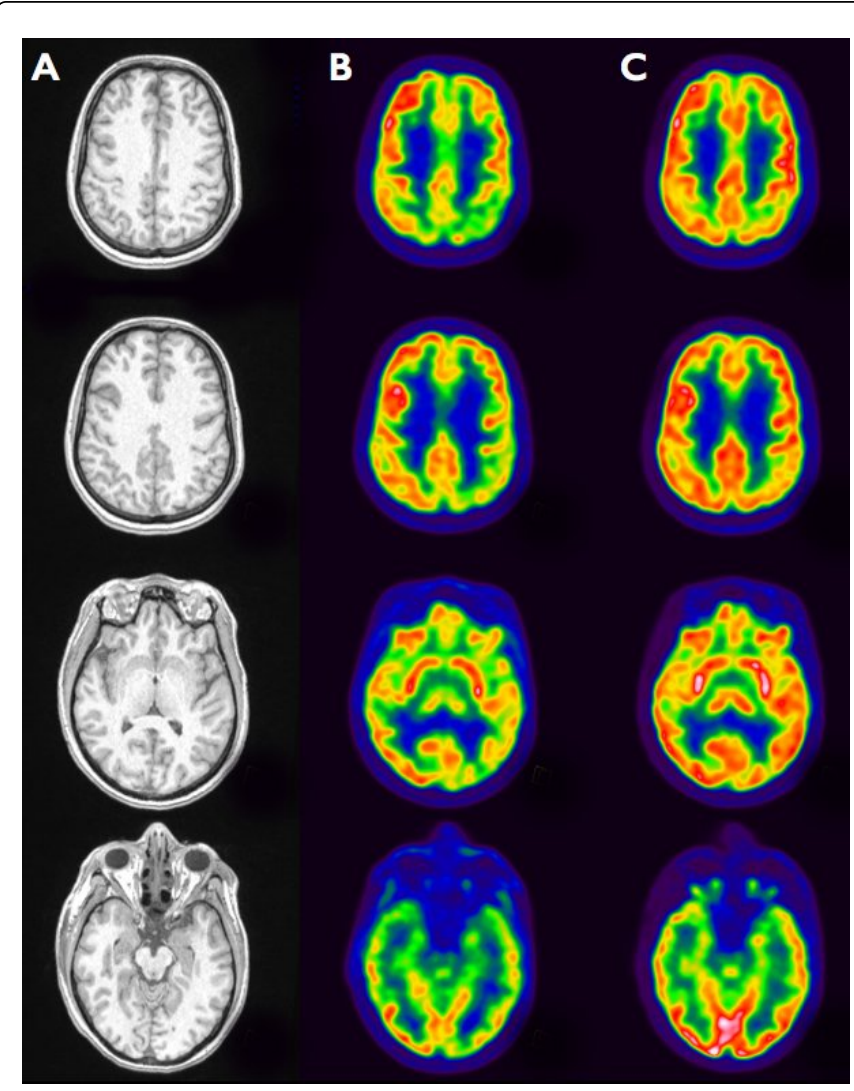

Figure 2: Matched transverse slices showing multimodal brain imaging: T1-weighted MRI (A), FDG PET during (B) and after (C) the hypoglycemic coma episode. During the coma episode (B) FDG PET showed hypometabolism of bilateral precuneus, left pre- and postcentral regions, left insula, left temporal regions and bilateral occipital regions (the patient's eyes were closed). After the coma episode (C), FDG PET showed a marked improvement of previously observed metabolic abnormalities as only the left temporal pole remained hypometabolic.

In conclusion, FDG PET and rs-fMRI are non-invasive imaging tools that are helpful in the assessment of consciousness levels and residual cognitive disorders in patients with metabolic severe coma. They both indirectly measure neuronal activity and have been recently used in a study to demonstrate differences in neuronal activity between commonly used mouse strains [14]. The detection of the DMN on rsfMRI and levels of cortical metabolism in frontal and parietal regions on FDG PET seem to be the most accurate markers of consciousness disorders when compared to currently available markers. Combining both methods with the development of PET/MRI may be more accurate and combine advantages of the two methods [15].

\section{References}

1. Laureys S, Schiff ND (2012) Coma and consciousness: paradigms (re)framed by neuroimaging. Neuroimage 61: 478-491.

2. Roquet DR, Pham BT, Foucher JR (2014) Manual selection of spontaneous activity maps derived from independent component analysis: criteria and inter-rater reliability study. J Neurosci Methods 223: 30-34.

3. Kalcher K, Huf W, Boubela RN, Filzmoser P, Pezawas L, et al. (2012) Fully exploratory network independent component analysis of the 1000 functional connectomes database. Front Hum Neurosci 6: 301.

4. Di Perri C, Stender J, Laureys S, Gosseries O (2014) Functional neuroanatomy of disorders of consciousness. Epilepsy Behav 30: 28-32.

5. Vanhaudenhuyse A, Noirhomme Q, Tshibanda LJ, Bruno MA, Boveroux P, et al. (2010) Default network connectivity reflects the level of consciousness in non-communicative brain-damaged patients. Brain 133: 161-171.

6. Shulman RG, Hyder F, Rothman DL (2014) Insights from neuroenergetics into the interpretation of functional neuroimaging: an alternative empirical model for studying the brain's support of behavior. J Cereb Blood Flow Metab 34: 1721-1735.

7. Sokoloff L (1977) Relation between physiological function and energy metabolism in the central nervous system. J Neurochem 29: 13-26.

8. Dietemann S, Noblet V, Imperiale A, Blondet C, Namer IJ (2015) FDG PET findings of the brain in sudden blindness caused by bilateral central retinal artery occlusion revealing giant cell arteritis. Clin Nucl Med 40: 45-46.

9. Fox PT, Raichle ME, Mintun MA, Dence C (1988) Nonoxidative glucose consumption during focal physiologic neural activity. Science 241: 462-464.

10. Attwell D, Laughlin SB (2001) An energy budget for signaling in the grey matter of the brain. J Cereb Blood Flow Metab 21: 1133-1145.

11. Stender J, Gosseries O, Bruno MA, Charland-Verville V, Vanhaudenhuyse A, et al. (2014) Diagnostic precision of PET imaging and functional MRI in disorders of consciousness: a clinical validation study. Lancet 384 : 514-522.

12. Thibaut A, Bruno MA, Chatelle C, Gosseries O, Vanhaudenhuyse A, et al. (2012) Metabolic activity in external and internal awareness networks in severely brain-damaged patients. J Rehabil Med 44: 487-494.

13. Nakayama N, Okumura A, Shinoda J, Nakashima T, Iwama T (2006) Relationship between regional cerebral metabolism and consciousness disturbance in traumatic diffuse brain injury without large focal lesions: an FDG-PET study with statistical parametric mapping analysis. J Neurol Neurosurg Psychiatry 77: 856-862.

14. Shah D, Deleye S, Verhoye M, Staelens S, Van der Linden A (2016) Resting-state functional MRI and [18F]-FDG PET demonstrate differences in neuronal activity between commonly used mouse strains. Neuroimage 125: 571-577.

15. Catana C, Drzezga A, Heiss WD, Rosen BR (2012) PET/MRI for neurologic applications. J Nucl Med 53: 1916-1925. 